\title{
New Evidence for High Activity of the Super-massive Black Hole in our Galaxy
}

\author{
M. Nobukawa, S. G. Ryu, T. G. Tsuru, and K. Koyama \\ Department of Physics, Graduate School of Science, Kyoto University, Sakyo-ku, Kyoto \\ 606-8502, Japan \\ nobukawa@cr.scphys.kyoto-u.ac.jp
}

\begin{abstract}
Prominent K-shell emission lines of neutral iron (hereafter, Fe I-K lines) and hard-continuum X-rays from molecular clouds (MCs) in the Sagittarius B (Sgr B) region were found in two separate Suzaku observations in 2005 and 2009. The Xray flux of the Fe I-K lines decreased in correlation with the hard-continuum flux by a factor of $0.4-0.5$ in four years, which is almost equal to the light-traveling across the MCs. The rapid and correlated time-variability, the equivalent width of the Fe I-K lines, and the K-edge absorption depth of Fe I are consistently explained by "X-ray echoes" due to the fluorescent and Thomson-scattering of an X-ray flare from an external source. The required flux of the X-ray flare depends on the distance to the MCs and its time duration. Even for a case with a minimum distance, the flux is larger than those of the brightest Galactic X-ray sources. Based on these facts, we conclude that the super-massive black hole Sgr $\mathrm{A}^{*}$ exhibited a large-flare a few hundred years ago with a luminosity of more than $4 \times 10^{39} \mathrm{erg} \mathrm{s}^{-1}$. The "X-ray echo" from Sgr B, located a few hundred light-years from Sgr $\mathrm{A}^{*}$, has now reached at the Earth.
\end{abstract}

Subject headings: stars: individual (Sagittarius A*) — Galaxy: center — ISM: clouds - ISM: abundances

\section{Introduction}

One of the most significant topics of research is X-rays emission in the Galactic center (GC) region, particularly the K-shell emission lines of neutral iron (hereafter, the Fe I-K lines) from the giant molecular clouds (MCs: Tsuboi et al. 1999) in Sagittarius B (Sgr B), located at $l=0.7^{\circ}$ on the Galactic plane. X-rays and the Fe I K lines from Sgr B were 
first detected by the Granat/ART-P telescope (Sunvaev et al. 1993) and the ASCA satellite (Koyama et al. 1996), respectively. The MCs are extremely cold, and hence cannot self-excite neutral irons to a higher level in order to emit the Fe I-K lines. The origin of Fe I-K lines has been a mystery. From laboratory experiments, two plausible origins have been proposed: the fluorescence after inner-shell ionization by the injection of electrons or emission of Xrays with energies greater than $7.1 \mathrm{keV}$ (the minimum ionization energy of Fe I). The X-ray spectra of the MCs, which show a strong absorption edge at $7.1 \mathrm{keV}$ together with intense Fe I-K $\alpha$ (L- to K-shell transition) and Fe I-K $\beta$ (M- to K-shell transition) lines, support the X-ray origin rather than the electron origin (Koyama et al. 1996; Murakami et al. 2000).

The X-ray origin was established by the recent discovery of rapid (order of several years) time-variability of Fe I-K lines (Koyama et al. 2008; Inui et al. 2009), and from the fact that only photons can produce such rapid variability in the MCs with size of the order of 10 light-years. Increase in the rapid time variability of the Fe I-K lines from other MCs near the GC has been discovered (Ponti et al. 2010). This suggests that the MCs have only been illuminated by an external X-ray source. Furthermore, time variability in the continuum Xray was found by Muno et al. (2007). In the X-ray fluorescent origin, Thomson scattered Xrays are also predicted in the hard X-ray band. In fact, the X-rays detected by Sunyaev et al. (1993) were the Thomson scattered component. Terrier et al. (2010) discovered time variable hard X-rays (20-60 keV) originating from the Sgr B2 region. In addition, Muno et al. (2007) found flickering continuum X-ray emissions from small clouds near the GC. Accordingly, X-rays from the clouds may be called "X-ray echoes." Fe I-K lines and hard continuum $\mathrm{X}$-rays are due to fluorescence and the Thomson scattering of external X-rays, respectively (Koyama et al. 1996; Murakami et al. 2000; Terrier et al. 2010). The "X-ray echoes" can easily produce apparent superluminal propagation, as Ponti et al. (2010) found in the MCs near the GC (Sunyaev \& Churazov 1998).

The X-ray luminosity of these clouds is $\sim 10^{35} \mathrm{erg} \mathrm{s}^{-1}$, and hence an illuminating object should be much brighter (>10 ${ }^{39} \mathrm{erg} \mathrm{s}^{-1}$; Sunyaev \& Churazov 1998; Murakami et al. 2000). One proposed scenario is that Sgr A*, the super-massive black hole with around four million solar masses in the GC located at a distance of several hundred light-years from the MCs (Ghez et al. 2008), was bright several hundred years ago with an X-ray luminosity millions times higher than the present luminosity of $10^{33}-10^{34} \mathrm{erg} \mathrm{s}^{-1}$ (Baganoff et al. 2001). The increase and decrease in the X-ray emission from the MCs may suggest intermittent activities of Sgr A* several hundred years ago (Inui et al. 2009; Ponti et al. 2010).

However, because the required luminosity of the external X-ray source depends on the distance to the MCs, the duration of X-ray irradiation, and the size and density of the MCs, the currently available data does not exclude the possibility of intense X-ray flares from near- 
by transient sources. Recently, Ponti et al. (2010) discovered superluminal motion in the Fe I-K emission from the MCs near the $\mathrm{GC}(l, b) \sim\left(0^{\circ} .1,0^{\circ} .1\right)$ and estimated that a large luminosity of $>1.3 \times 10^{38} \mathrm{erg} \mathrm{s}^{-1}$, which is almost equal to the Eddington luminosity for a stellar mass object was required for illumination. They concluded that the past flare of Sgr $\mathrm{A}^{*}$ had illuminated the MCs. However, for other MCs emitting Fe I K, particularly, those in Sgr B, there is no clear evidence that $\mathrm{Sgr} \mathrm{A}^{*}$ was the only candidate for their illumination source. This paper provides clear evidence for the scenario of a past large-flare of Sgr A*.

The errors in the estimation in this paper are at the $1 \sigma$ confidence level. The distance to the GC is assumed to be the canonical value of 26,000 light-years (Eisenhauer et al. 2003; Ghez et al. 2008). Because we use the Galactic coordinate system, we define "east" and "west" as the positive and negative Galactic longitude sides, respectively.

\section{Observation and Data Reduction}

To identify the external X-ray source for the "X-ray echo," Sgr B2 would be the best target because (i) it is one of the brightest X-ray echo sources, (ii) its three-dimensional position is well determined (Reid et al. 2009; Rvu et al. 2009), and (iii) the Galactic center diffuse X-ray emission (GCDX: Koyama et al. 1996) is relatively small and uniform. Therefore, possible contamination from the GCDX can be reliably subtracted.

We observed the Sgr B region with the X-ray CCD cameras the X-ray Imaging Spectrometer (XIS; Koyama et al. 2007a) aboard the Suzaku satellite (Mitsuda et al. 2007). To examine time variability, the observations were performed twice in October 2005 and September 2009, at identical pointing positions within $4^{\prime \prime}$. The sequence numbers for the two observations are 100037060 and 504004020. The effective exposure times in 2005 and 2009 were $21.3 \mathrm{~h}$ and $56.1 \mathrm{~h}$, respectively. Four XIS (XIS0, 1, 2, and 3) and three XIS (XIS0, 1, and 3) cameras were available in 2005 and 2009, respectively. We discarded the data obtained in a one-fourth field (segment A) of XIS0 for both observations because it has become nonfunctional since June 2008.

Although the effective energy range of XIS is $0.2-10 \mathrm{keV}$, we used the $4-10 \mathrm{keV}$ band data for the spectral analysis. This is because soft X-rays from the target (Sgr B) are absorbed by the interstellar medium toward the GC region. We obtained the data for non-

X-ray background (NXB) caused by the cosmic-rays using xisnxbgen (Tawa et al. 2008) and then subtracted the NXB from the observed data. We then corrected the local differences of the effective area for the data analysis (the vignetting correction). 


\section{Analysis}

\subsection{X-ray Images}

Figure 1a shows the X-ray images for the Fe I-K $\alpha(6.4 \mathrm{keV})$ line of the Sgr B region in 2005, where bright diffuse sources are clearly detected (Kovama et al. 2007b). According to a previous work (Koyama et al. 2007b), we hereafter refer to the two sources as Sgr B2 and M 0.74-0.09. Figure 1b shows the same image taken four years later in 2009. Comparing the two images, we observe that the two bright sources have darkened in the four years, whereas there is no significant change in the brightness of the surrounding region.

In addition, Figure 1c and 1d show the hard X-ray images of the Sgr B region in the 8-10 keV band for the 2005 and 2009 observations, respectively. As shown in Figure 1k, hard X-ray excesses at the positions of Sgr B2 and M 0.74-0.09 in 2005 are observed. This is the first resolved hard X-ray image of Sgr B2 and M 0.74-0.09, although in the past X-ray observation, the hard X-ray excess near Sgr B2 has been found with an insufficient special resolution of $12^{\prime}$ (Terrier et al. 2010). On the other hand, the hard X-ray fluxes from these MCs are almost comparable to those of the surrounding areas and hence no significant hard X-ray excesses are found in the 2009 data (Figure 1d). The significance levels of the hard Xrays from Sgr B2 and M 0.74-0.09 with respect to the background region shown by the white dashed line are 5 and $4 \sigma$ in 2005 and $<3 \sigma$ in 2009, respectively. These facts suggest that the hard X-rays from Sgr B2 and M 0.74-0.09 decreased from 2005 to 2009 in correlation with the decrease in the Fe I-K fluxes.

\subsection{Spectra}

We investigate the X-ray spectra to study the time variability more quantitatively. The regions selected for the Sgr B2 and M 0.74-0.09 spectra are indicated with the white solid lines in Figure 1. The background region is also shown with white dashed lines, where the dashed small circle in which an MC might be emitting faint Fe I-K is excluded. Figures 2a and $2 \mathrm{~b}$ are the background-subtracted spectra of Sgr B2 and M 0.74-0.09, respectively. The spectra in 2005 and 2009 are shown by black and red crosses, respectively. As seen in both spectra, the fluxes of the Fe I-K $\alpha$ lines and the hard continuum have simultaneously decreased from 2005 to 2009.

We fit the spectra with a phenomenological model of an absorbed power-law and the Fe I-K $\alpha$ and Fe I-K $\beta$ lines at $6.4 \mathrm{keV}$ and $7.06 \mathrm{keV}$, respectively. A supernova remnants candidate G 0.61+0.01 (Kovama et al. 2007b) is located 4' away from Sgr B2 and may slightly 
Table 1: Best-fit parameters ${ }^{\mathrm{a}}$.

\begin{tabular}{lllll}
\hline Source (Year) & $N_{\mathrm{H}}$ & Fe I K $\alpha$ & Fe I K $\beta$ & $5-10 \mathrm{keV}$ \\
unit & $10^{23} \mathrm{~cm}^{-2}$ & $10^{-5 \mathrm{~b}}$ & $10^{-5 \mathrm{~b}}$ & $10^{-12 \mathrm{c}}$ \\
\hline Sgr B2 (2005) & $8.4 \pm 0.9$ & $13.6 \pm 0.7$ & $1.3 \pm 0.3$ & $4.3 \pm 0.5$ \\
Sgr B2 (2009) & $8.8 \pm 1.4$ & $5.2 \pm 0.5$ & $<0.4$ & $2.1 \pm 0.3$ \\
M 0.74-0.09 (2005) & $5.7 \pm 0.4$ & $5.1 \pm 0.4$ & $0.4 \pm 0.2$ & $1.7 \pm 0.2$ \\
M 0.74-0.09 (2009) & $6.5 \pm 1.2$ & $2.7 \pm 0.3$ & $0.5 \pm 0.2$ & $0.9 \pm 0.2$ \\
\hline
\end{tabular}

${ }^{a}$ The error ranges in this table are calculated at the $1 \sigma$ confidence level.

${ }^{b}$ Observed flux in the unit of photons $\mathrm{s}^{-1} \mathrm{~cm}^{-2}$.

${ }^{c}$ Observed flux in the unit of ergs s${ }^{-1} \mathrm{~cm}^{-2}$.

contaminate the Sgr B2 spectrum. Therefore, we consider the contamination of the thermal plasma of G $0.61+0.01$ by adding a plasma model (Mazzotta et al. 1998) with a fixed temperature of 37 million $\mathrm{K}$ and an iron abundance of $Z_{\mathrm{Fe}}=5.1$ solar (Koyama et al. 2007b). The contamination is then found to be less than $10 \%$, and hence the error due to the possible uncertainty of flux contamination can be neglected.

The four spectra (Sgr B2 and M 0.74-0.09 for 2005 and 2009) are simultaneously fitted with the free parameters of the absorption column density $N_{\mathrm{H}}$, the iron abundance $Z_{\mathrm{Fe}}$, the photon index $\Gamma$, and the fluxes of the continuum and the Fe I-K lines. Among them, only $Z_{\mathrm{Fe}}$ and $\Gamma$ are set to be common for all spectra. The fit is acceptable with $\mathrm{chi}^{2} /$ d.o.f. of $216 / 217$ (null hypothesis probability $=0.6$ ). The best-fit common parameters of $Z_{\mathrm{Fe}}$ and $\Gamma$ are $1.3 \pm 0.3$ solar and $2.5 \pm 0.6$ solar, respectively, and those of the other free parameters are listed in Table 1.

We find that the fluxes of the Fe I-K $\alpha$ lines and the hard continuum band for Sgr B2 decrease by a factor of $0.39 \pm 0.04$ and $0.49 \pm 0.09$ from 2005 to 2009 , respectively. These variation factors are consistent within the $1 \sigma$ confidence level with the mean value of $0.41 \pm 0.04$. The variation factors of $0.53 \pm 0.07$ (Fe I-K $\alpha$ lines) and $0.53 \pm 0.13$ (continuum band) for M 0.74-0.09 are also consistent with the mean value of $0.53 \pm 0.06$.

\section{Discussion}

On the basis of the 2005 data, we confirmed that the two MCs (Sgr B2 and M 0.74-0.09) exhibit intense Fe I-K lines. In addition, we resolved the hard continuum emission from Sgr B2 detected by Granat and INTEGRAL (Sunyaev et al. 1993; Revnivtsev et al. 2004) into 
emissions from two MCs. The most important discovery is that the correlated decrease in the four years between the Fe I-K lines and the hard continuum is synchronized in the two MCs, although Terrier et al. (2010) reported a correlated decrease in the entire Sgr B region. Because the data for these results are obtained with the same instrument (Suzaku/XIS) and an identical pointing field, the possible systematic errors are minimized. The correlation of the variability between the two MCs and the two components (Fe I-K lines and hard continuum) indicate that they are of common origin.

As we have already discussed, the origin is likely to be X-ray irradiation by an external source ("X-ray echo"). Then, the equivalent width of the Fe I-K $\alpha$ lines $\left(\mathrm{EW}_{6.4 \mathrm{keV}}\right)$ is expressed as $1.0 \times\left(Z_{\mathrm{Fe}} / 1\right.$ solar) keV (Tatischeff 2003; Nobukawa et al. 2010). As the observed $\mathrm{EW}_{6.4 \mathrm{keV}}$ is $1.0-1.5 \mathrm{keV}$, we derive the iron abundance as $Z_{\mathrm{Fe}}=1.0-1.5$ solar. The absorption column density $N_{\mathrm{H}}$ of about $10^{24} \mathrm{~cm}^{-2}$ is significantly larger than the value of $10^{23} \mathrm{~cm}^{-2}$ for typical sources in the GC (Muno et al. 2004), and hence, the major fraction would be owing to the absorption in the MCs. The iron absorption edge depth at $7.1 \mathrm{keV}$ gives the iron abundance in the MCs of $Z_{\mathrm{Fe}}=1.3 \pm 0.3$ solar, which is consistent with that derived from $\mathrm{EW}_{6.4 \mathrm{keV}}$. These facts firmly support the "X-ray echo" for the X-ray emission from Sgr B2 and M 0.74-0.09: the fluorescent Fe I-K line and the Thomson-scattered hard-continuum.

We found that the fluxes of Sgr B2 and M 0.74-0.09 had reduced by factors of $0.41 \pm 0.4$ and $0.53 \pm 0.6$ during the four years, respectively. This result shows that the decrease in Fe I-K emission from 2000 to 2005 found by previous works (Koyama et al. 2008; Inui et al. 2009) had continued at least until 2009. Inui et al. (2009) reported that the decrease rate of the Fe I-K emission is $~ 0.6$ in five years (2000-2005). Thus, the X-ray fluxes had decreased by a factor of $\sim 0.2$ during the decade. In addition, Terrier et al. (2010) showed a decay of up to 0.4 in a hard X-ray flux from 2003 to 2009, which is almost consistent with our result for the hard continuum.

The schematic view of the "X-ray echo" scenario is shown in Figure 3. As the incident $\mathrm{X}$-ray flare moves at light speed in the MCs, the echo spot also moves at light speed. Because the density of the MCs is not uniform, the brightness of the X-ray echoes would be higher in high-density regions than in low-density regions. The X-ray echoes in 2005 was produced in high-density regions and then moved into low-density regions in 2009. Ponti et al. (2010) proposed that the flare lasted for a large duration of $\sim 300$ years (long flare). In the long flare scenario, is was suggested that the MCs in the Sgr B2 region were illuminated by Xrays at the end of the long flare. On the other hand, Yu et al. (2011) indicated that a single shorter flare can explain the X-ray light curve of Sgr B2. We do not yet have enough data to determine the probable scenario.

The required luminosity $\left(L_{\mathrm{X}}\right)$ of the X-ray flare to produce the "X-ray echo" of the MCs 
depends on the time duration $(t)$ of the flare, the distances $(d)$ to the MCs, and the optical depths $(\tau)$ along the X-ray pass band in the MCs. In particular, $L_{\mathrm{X}}$ also depends on the density profiles and the sizes of MCs. The MC sizes may be $\sim 10$ light years, almost equal to those in the selected regions shown in Figure 1. Using the observed $N_{\mathrm{H}}$ of about $10^{24} \mathrm{~cm}^{-2}$, $\tau$ is estimated to be about 0.1 . Then, for the two cases of long flare $t>10$ years and short flare $t<10$ years, we can estimate $L_{\mathrm{X}}$ from Sunyaev \& Churazov (1998) as follows:

$$
L_{\mathrm{X}}= \begin{cases}4 \times 10^{39}\left(\frac{t}{10 \text { year }}\right)^{-1}\left(\frac{d}{500 \mathrm{ly}}\right)^{2} \operatorname{erg~s}^{-1} & (t \leq 10 \text { year }) \\ 4 \times 10^{39}\left(\frac{d}{500 \mathrm{ly}}\right)^{2} \text { erg s }^{-1} & (t>10 \text { year. })\end{cases}
$$

Using radio observations, Murakami et al. (2000) indicated that the X-ray peak shifted from the MC core to the east direction by $\sim 1 . ' 2$; therefore, the X-ray flare source should be located on the west side of Sgr B2. Moreover, the minimum value of the required luminosity is given when the flare-source is near the west-rim of Sgr B2. In this case, the distance $d$ to the other MC, M $0.74-0.09$ is about $0.1^{\circ}$ or 50 light-years (Figure 1). The minimum value of the required luminosity is $L_{\mathrm{X}}=4 \times 10^{37} \mathrm{erg} \mathrm{s}^{-1}$. As Equation (1) is derived from simple assumptions and approximations, some uncertainties exist in $L_{\mathrm{X}}=4 \times 10^{37} \mathrm{erg} \mathrm{s}^{-1}$, but the errors should not be larger than one order of magnitude.

The most conservative value of $L_{\mathrm{X}}$ is $4 \times 10^{36} \mathrm{erg} \mathrm{s}^{-1}$, which is almost equal to that of the brightest X-ray sources in our Galaxy. Because we did not find such stable sources in the GC, one possible candidate is a transient X-ray binary system. X-ray monitoring in the GC has been conducted since almost 10 years with the INTEGRAL satellite (Kuulkers et al. 2007). All bright transient sources exhibited peak luminosities lower than a few times of $10^{37} \mathrm{erg} \mathrm{s}^{-1}$ (Sakano et al. 2002; Werner et al. 2003). Moreover, the flare durations $(t)$ are shorter than 0.1 years (Sakano et al. 2002; Werner et al. 2003). The average luminosity of the transient sources over 10 years is a few times of $10^{35} \mathrm{erg} \mathrm{s}^{-1}$, which is 10 times lower than the minimum required luminosity by the most conservative estimation.

These facts indicate that the transient binary system is an unlikely candidate for the irradiation source. Therefore, we conclude that the large-flare of Sgr A*, the super-massive black hole, is the irradiation source. The large flare with a luminosity of more than $4 \times$ $10^{39} \mathrm{erg} \mathrm{s}^{-1}$ might have occurred a few hundred years ago. At present, the X-ray luminosity of Sgr A* is about $10^{34} \mathrm{erg} \mathrm{s}^{-1}$ and it exhibits numerous flares. However, the flare scales are relatively small and their luminosity can at most reach 180 times that of the quiescent level (Porquet et al. 2003). The large flare predicted in this study, which is more than $10^{4}$ times the largest flare observed until now, would be new evidence for past high activity of the super-massive black hole at the center of our Galaxy. 
This work is supported by the Grant-in-Aid for the Global COE Program "The Next Generation of Physics, Spun from Universality and Emergence" from the Ministry of Education, Culture, Sports, Science and Technology (MEXT) of Japan. This work is also supported by Grant-in-Aids from the Ministry of Education, Culture, Sports, Science and Technology (MEXT) of Japan, Scientific Research A, No. 18204015 (KK), and Scientific Research B, No. 20340043 (TT). MN and SGR are supported by JSPS Research Fellowship for Young Scientists.

\section{REFERENCES}

Baganoff, F. K., et al. 2001, Nature, 413, 45

Eisenhauer, F., et al. 2003, ApJ, 597, L121

Ghez, A. M., et al. 2008, ApJ, 689, 1044

Inui, T., Koyama, K., Matsumoto, H., \& Tsuru, T. G. 2009, PASJ, 61, S241

Koyama, K., Maeda, Y., Sonobe, T., Takeshima, T., Tanaka, Y., \& Yamauchi, S. 1996, PASJ, 48, 249

Koyama, K., et al. 2007a, PASJ, 59, S23

Koyama, K., et al. 2007b, PASJ, 59, S221

Koyama, K., Inui, T., Matsumoto, H., \& Tsuru, T. G. 2008, PASJ, 60, S201

Kuulkers, E., et al. 2007, A\&A, 466, 595

Mazzotta, P., Mazzitelli, G., Colafrancesco, S., \& Vittorio, N. 1998, A\&AS, 133, 403

Mitsuda, K., et al. 2007, PASJ, 59, S1

Muno, M. P., et al. 2004, ApJ, 613, 326

Muno, M. P., Baganoff, F. K., Brandt, W. N., Park, S., \& Morris, M. R. 2007, ApJ, 656, L69

Murakami, H., Koyama, K., Sakano, M., Tsujimoto, M., \& Maeda, Y. 2000, ApJ, 534, 283

Nobukawa, M., Koyama, K., Ryu, S. G., Tsuru, T. G., \& Tatischeff, V. 2010, PASJ, 62, 423

Ponti, G., Terrier, R., Goldwurm, A., Belanger, G., \& Trap, G. 2010, ApJ, 714, 732 
Porquet, D., Predehl, P., Aschenbach, B., Grosso, N., Goldwurm, A., Goldoni, P., Warwick, R. S., \& Decourchelle, A. 2003, A\&A, 407, L17

Reid, M. J., Menten, K. M., Zheng, X. W., Brunthaler, A., \& Xu, Y. 2009, ApJ, 705, 1548

Revnivtsev, M. G., Churazov, E. M., Sazonov, S. Yu., Sunyaev, R. A., Lutovinov, A. A., Gilfanov, M. R., Vikhlinin, A. A., Shtykovsky, P. E., \& Pavlinsky, M. N. 2004, A\&A, 425, L49

Ryu, S. G., Koyama, K., Nobukawa, M., Fukuoka, R., \& Tsuru, T. G. 2009, PASJ, 61, 751

Sakano, M., Koyama, K., Murakami, H., Maeda, Y., \& Yamauchi, S. 2002, ApJS, 138, 19

Sunyaev, R., Markevitch, M., \& Pavlinsky, M. 1993, ApJ, 407, 606

Sunyaev, R., \& Churazov, E. 1998, MNRAS, 297, 1279

Tsuboi, M., Handa, T., \& Ukita, N. 1999, ApJS, 120, S1

Terrier, R., et al. 2010, ApJ, 719, 143

Tatischeff, V. 2003, in Final Stage of Stellar Evolution, ed. C. Motch \& J. M. Hameury (Les Ulis: EDP Sciences), 79

Tawa, N., et al. 2008, PASJ, 60, S11

Werner, N., et al. 2003, A\&A, 416, 311

Yu, Yun-Wei, Cheng, K. S., Chernyshov, D. O., \& Dogiel, V. A. 2011, MNRAS, 411, 2002 

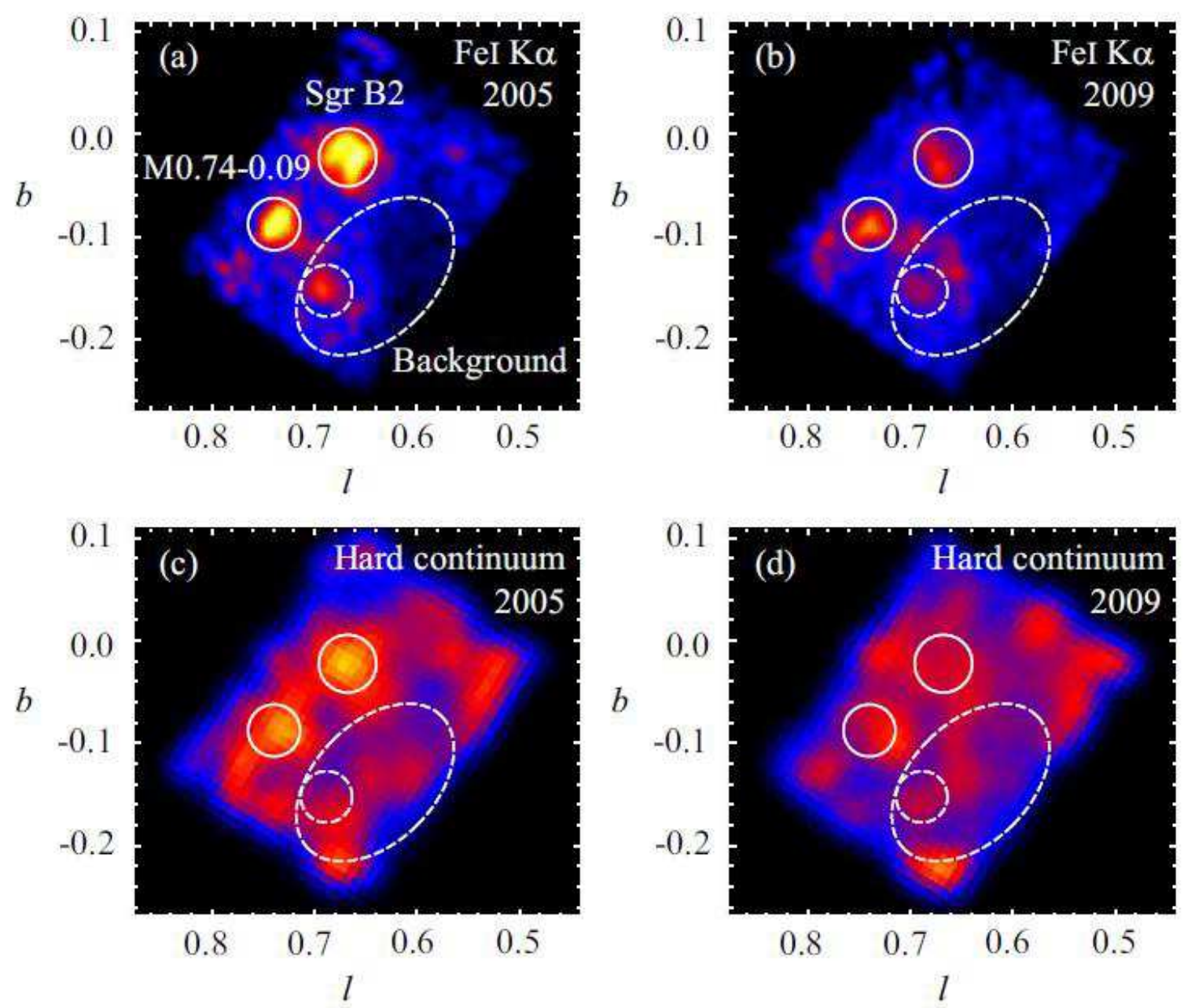

0

Fig. 1.- X-ray images for Sgr B2 and M0.74-0.09 in the field of view of $17.8^{\prime} \times 13.4^{\prime}$. The vertical and horizontal axes are the Galactic longitude $(l)$ and latitude $(b)$ in degrees $\left(^{\circ}\right)$, respectively. (a): The Fe I-K $\alpha(6.4 \mathrm{keV})$ band images in 2005. The bin size is 8.3" and is smoothed with a Gaussian kernel of $1 \sigma=42^{\prime \prime}$. (b): The same as (a) but in 2009. (c): The hard continuum $(8-10 \mathrm{keV})$ band images in 2005. The bin size is 33.3 " and is smoothed with a Gaussian kernel of $1 \sigma=100^{\prime \prime}$. (d) The same as (c) but in 2009. The source regions for Sgr B2 and M 0.74-0.09 are shown in the white solid circles, centered at $(l, b)$ $=\left(0.6676^{\circ},-0.0226^{\circ}\right)$ with a radius of $1.7^{\prime}$ and at $(l, b)=\left(0.7394^{\circ},-0.087^{\circ}\right)$ with a radius $=1.5^{\prime}$, respectively. The background region is shown by the white dashed ellipse centered at $(l, b)=\left(0.6410^{\circ},-0.1523^{\circ}\right)$ with the major and minor radii of $5.5^{\prime}$ and $3.5^{\prime}$, respectively. The inner white dashed circle at $(l, b)=\left(0.6885^{\circ},-0.1523^{\circ}\right)$ with a radius $=1.5^{\prime}$ is excluded for the background region (see text). In this figure, $0.1^{\circ}\left(6^{\prime}\right)$ corresponds to 50 light-years. The color scale is arbitrarily adjusted for comparison. 

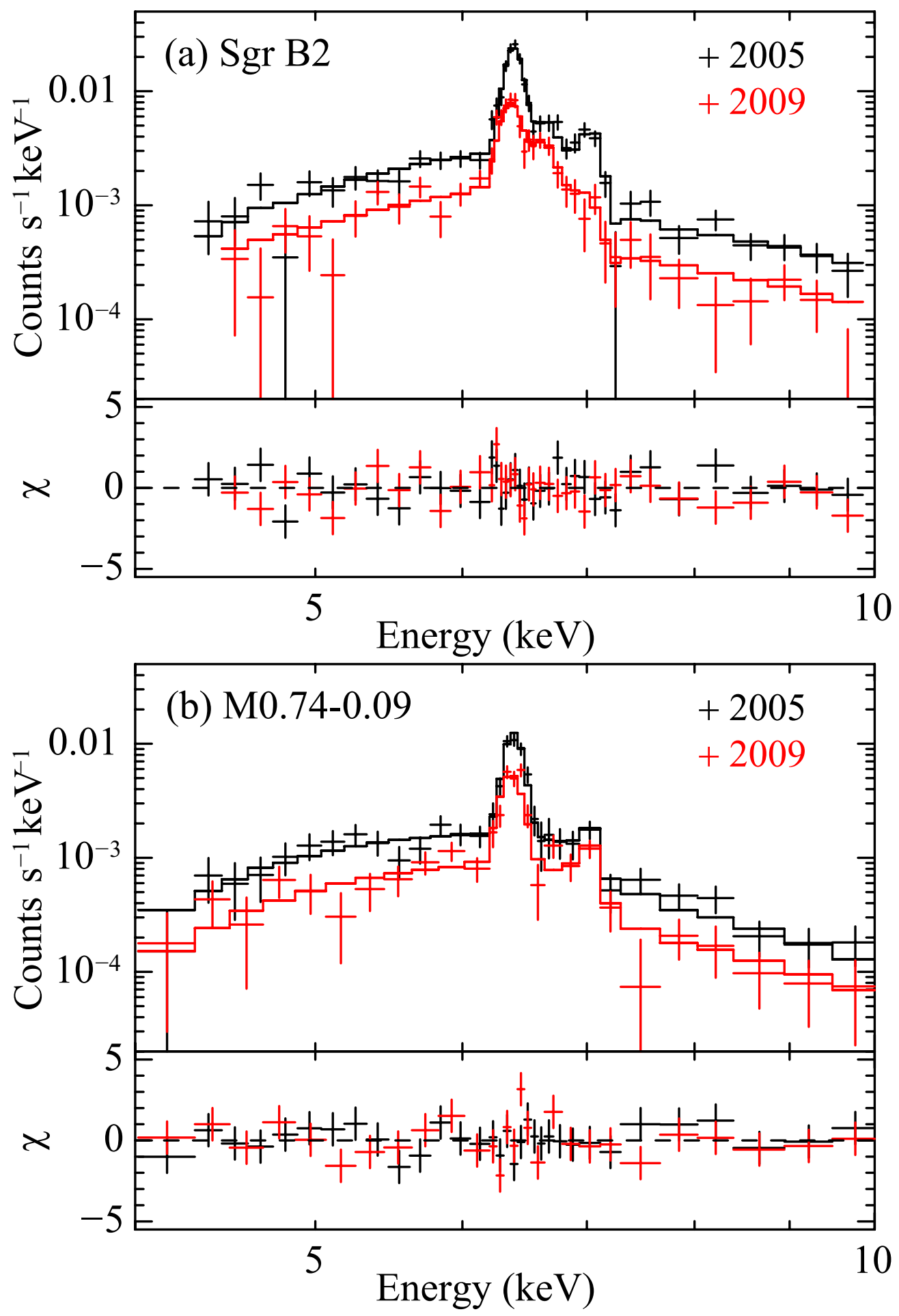

Fig. 2.- X-ray spectra of Sgr B2 (a) and M 0.74-0.09 (b). Spectra of the 2005 observation and the 2009 observation are indicated with black and red crosses, respectively. The bestfit models are shown with solid histograms. Residuals between the observed data and the models are shown in the bottom panels of (a) and (b). 


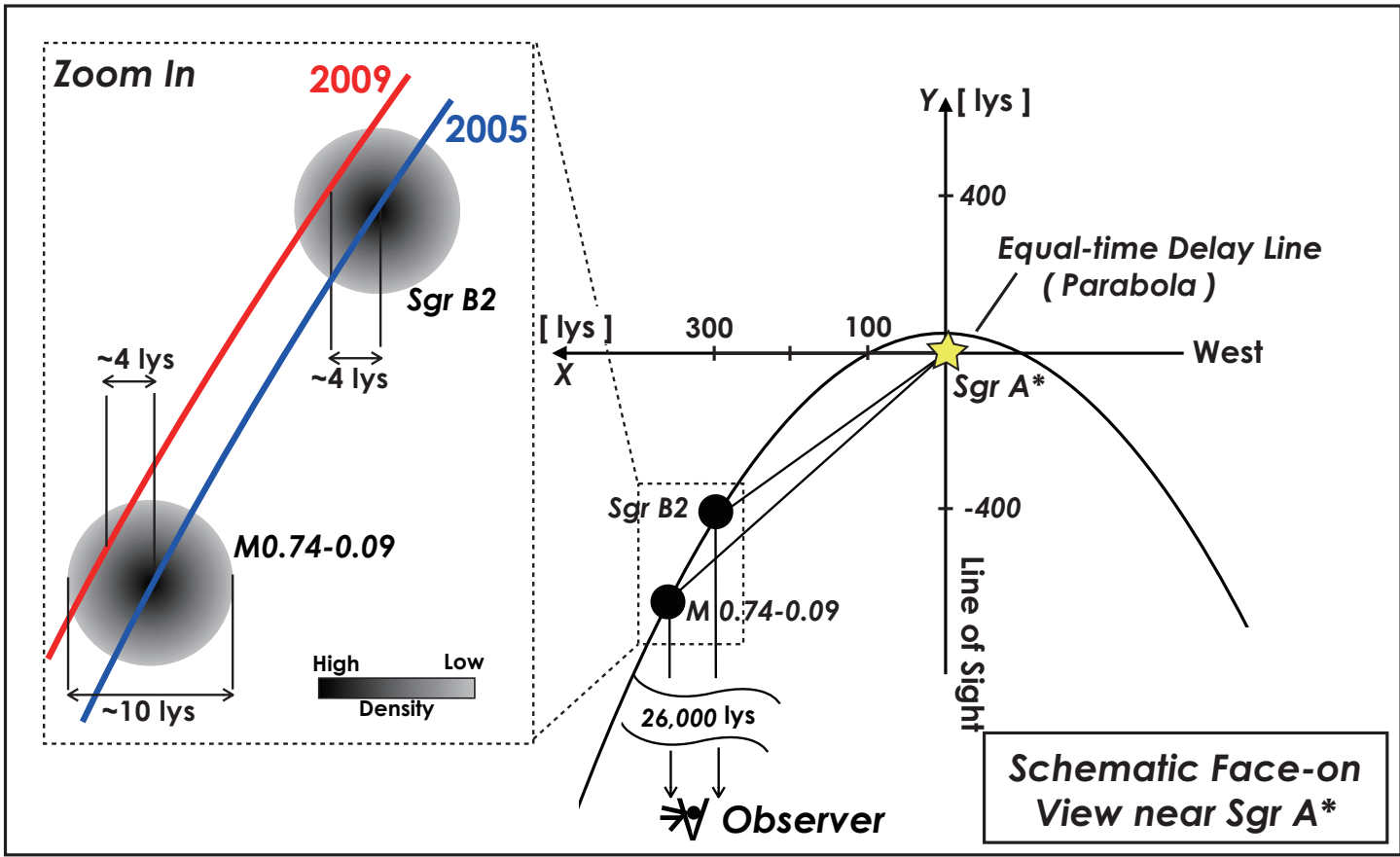

Fig. 3.- Schematic face-on view of the movement of the X-ray echoes in the molecular clouds. The irradiating source, the super-massive black hole Sgr A*, is indicated with the yellow star. The line-of-sight positions of Sgr B2 and M 0.74-0.09 are adopted from the results of a previous work (Reid et al. 2009). The solid parabola guides the eyes to see the X-ray echoes from Sgr B2 and M 0.74-0.09 arriving at the observer at the same time (equal-time delay line). The unit of distance is light-years (lys). 